\title{
EFICIENCIA DE LEVADURAS PARA LA CRÍA MASIVA DE ANASTREPHA LUDENS, $A$. OBLIQUA Y CERATITIS CAPITATA (DIPTERA: TEPHRITIDAE)
}

\author{
YEAST EFFICACY FOR MASS REARING OF ANASTREPHA LUDENS, A. OBLIQUA, AND \\ CERATITIS CAPITATA (DIPTERA: TEPHRITIDAE)
}

\section{Emilio HernándeZ,,${ }^{1, *}$ Pedro RIVERA, ${ }^{1}$ MARYsol ACEITUNO-MEdiNA, ${ }^{1}$ Reynaldo AGUILAR- LAPARRA, ${ }^{1}$ LUIS QUINTERO-FONG ${ }^{1}$ Y DINA OROZCO-DÁVILA ${ }^{1}$}

\author{
${ }^{1}$ Programa Moscafrut SAGARPA-IICA. Subdirección de Desarrollo de Métodos. Camino a los Cacahotales S/N, \\ Metapa de Domínguez, Chiapas C. P. 30860. México. \\ * Autor corresponsal: emilio.hernandez@iica-moscafrut.org.mx
}

Recibido: 26/01/2015; aceptado: 04/08/2016

Editor responsable: Vicente Hernández Ortiz

\begin{abstract}
Hernández, E., Rivera, P., Aceituno-Medina, M., Aguilar-Laparra, R., Quintero-Fong L., \& Orozco-Dávila, D. (2016). Eficiencia de levaduras para la cría masiva de Anastrepha ludens, $A$. obliqua y Ceratitis capitata (Diptera: Tephritidae). Acta Zoológica Mexicana (n.s.), 32(3), 240-252.
\end{abstract}

RESUMEN. La levadura inactiva en polvo es el único ingrediente disponible como fuente de proteína, en las dietas larvarias para la cría masiva de todas las especies de moscas de la fruta, proporcionando una proteína de alto valor biológico, vitaminas del complejo B, carbohidratos y grasas esenciales. Además, es el ingrediente con mayor costo, por lo cual se buscan continuamente alternativas de bajo costo. Desde el establecimiento de la cría masiva de A. ludens en 1994 y de A. obliqua en 1997 en la Planta de Metapa (México), se ha utilizado levadura tipo torula de una sola marca comercial, lo cual genera una dependencia. Las levaduras secas inactivas existentes en el mercado, de acuerdo con su composición tienen potencial para ser utilizadas en la preparación de dietas para la producción masiva de moscas de la fruta. Por ello, el objetivo de este trabajo fue determinar si los parámetros fisicoquímicos y microbiológicos que describen la composición de las levaduras permiten evaluar su eficiencia, mediante el establecimiento de un bioensayo a nivel experimental y semi-masivo para la preparación de dietas para la cría de larvas de moscas de la fruta. Se evaluaron seis levaduras comerciales para la cría y desarrollo de larvas de Anastrepha ludens, A. obliqua y Ceratitis capitata cepa Vienna 8 TSL sin inversión cromosómica, en las Plantas Moscafrut y Moscamed (SAGARPA-IICA) ubicadas en Metapa de Domínguez, Chiapas, México. Estas levaduras fueron seleccionadas con base en tres etapas: 1) Caracterización fisicoquímica y microbiológica de las levaduras. La caracterización fisicoquímica consistió en la determinación del contenido de humedad, $\mathrm{pH}$, acidez, solubilidad, densidad y contenido de proteína, mientras que la caracterización microbiológica consistió en determinar el contenido total de hongos, levaduras y coliformes. 2) Bioensayo a nivel experimental: se realizó inicialmente con las seis marcas comerciales evaluando el rendimiento y calidad de moscas producidas; y 3) Evaluación a nivel semi-masivo: De acuerdo con los parámetros de rendimiento se redujeron a solo tres marcas para las pruebas a nivel semi-masivo; Lake States, Lallemand LBI2163b
Hernández, E., Rivera, P., Aceituno-Medina, M., Aguilar-Laparra, R., Quintero-Fong L., \& Orozco-Dávila, D. (2016). Yeast efficacy for mass rearing of Anastrepha ludens, A. obliqua and Ceratitis capitata (Diptera: Tephritidae). Acta Zoológica Mexicana (n.s.), 32(3), 240-252.

ABSTRACT. The inactive yeast powder is the single ingredient available as sources of protein in the larval diets for mass rearing of all species of fruit flies, providing a protein of high biological value, $\mathrm{B}$ vitamins, carbohydrates and essential fats. In addition, it is the most expensive ingredient, thus we are continuously looking for low-cost alternatives. Since the establishment of mass rearing production of $A$. ludens in 1994, and A. obliqua in 1997, we have used torula yeast of a single trademark, which creates a dependency. Inactive dry yeasts available in the market, could be used potentially in the diet preparation for mass rearing of fruit flies. Therefore, the aim of this study was to determine whether the physicochemical and microbiological parameters describing the composition of yeasts are efficient, assessed through the establishment of experimental and semi-massive bioassays in the preparation of larval diet for mass rearing production. Six commercial yeasts for rearing and larval development were evaluated for Anastrepha ludens, A. obliqua and Ceratitis capitata strain Vienna 8 TSL without chromosomal inversion, in the Moscafrut and Moscamed facilities (SAGARPA-IICA) at Metapa de Domínguez (Chiapas, Mexico). Yeasts were selected on the basis on three stages: 1) the physical, chemical and microbiological characterization. The physicochemical traits consisted of determination of moisture content, $\mathrm{pH}$, acidity, solubility, density and protein content; while the microbiological characterization was assessed through total content of fungi, yeasts and coliforms. 2) Experimental bioassays. To evaluate the performance and quality of flies produced, initially six trademarks were used; and (3) Assessment to semi-massive scale. According to performance parameters, the semi-massive bioassays were conducted with three finalist brands: Lake States, Lallemand LBI2163b, and Nutribio for A. ludens and A. obliqua; and Lake States, Lallemand LBI2163b, and Nupro yeast extract were used for C. capitata. Prior to these bioassays, the content of vitamins and amino acids of the three finalist brands was determined. The results of the microbiological and 
y Nutribio para $A$. ludens y $A$. obliqua, mientras que para $C$. capitata fueron utilizadas Lake States, Lallemand LBI2163b y extracto de levadura Nupro. Previo a esta evaluación, se determinó el contenido de vitaminas y de aminoácidos a las tres marcas finalistas. Los resultados indicaron que los parámetros microbiológicos y físicoquímicos, incluyendo, la composición de aminoácidos, minerales y vitaminas de las levaduras, no permiten determinar la eficiencia de las levaduras para la cría masiva de Anastrepha ludens, A. obliqua y C. capitata. No obstante, las levaduras Nutribio y Lallemand LB12163b son una alternativa para la cría masiva de moscas de A. ludens y A. obliqua, respectivamente. Se sugiere que la evaluación de levaduras en la dieta larvaria requiere de un bioensayo a nivel experimental que incluya como variable respuesta la competencia sexual.

Palabras clave: dietas larvarias, fuente de proteína, técnica del insecto estéril, cría masiva.

\section{INTRODUCCIÓN}

Las moscas de la fruta (Diptera: Tephritidae) son de las plagas con mayor importancia económica en los países tropicales y subtropicales, causando grandes daños en la producción y en el comercio internacional de frutos frescos (Hendrichs, 1996). Es por ello que para su control se utiliza la Técnica del Insecto Estéril (TIE). Algunas especies para las cuales se aplica la TIE incluye a la mosca del Mediterráneo Ceratitis capitata (Wiedemann, 1824), la mosca mexicana de la fruta Anastrepha ludens (Loew, 1873), la mosca de la Indias Occidentales $A$. obliqua (Macquart, 1835) la mosca del Caribe A. suspensa (Loew, 1862), la mosca del melón Bactrocera cucurbitae (Coquillett, 1849), la mosca de Queensland B. tryoni (Froggatt, 1897), y algunas de las especies del complejo Bactrocera dorsalis (Cáceres et al., 2014). La TIE se aplica en México contra C. capitata desde 1979 (Hendrichs et al., 1983, Enkerlin, 2005); contra A. ludens desde 1994 (Reyes et al., 2000); y contra A. obliqua desde 2001 (Artiaga-López et al., 2004, Orozco et al., 2004).

Las Plantas Moscafrut y Moscamed para la cría y esterilización de moscas de la fruta, pertenecen a la Dirección General de Sanidad Vegetal (SENASICA - SAGARPA), se localizan en Metapa de Domínguez, Chiapas, México. La Planta Moscafrut tiene actualmente una meta semanal de producción de 175 y 50 millones de moscas estériles de A. ludens y A. obliqua, respectivamente, capaces de aparearse con moscas silvestres de la misma especie. Mientras que en la Planta Moscamed se producen 500 millones de machos estériles por semana de $C$. capitata cepa Vienna 8 TSL sin inversión cromosómica. Para llevar a cabo la cría masiva de estos insectos es primordial contar con una dieta que contenga nutrimentos que puedan ser asimilados por la larva, necesarios para su desarrollo, crecimiento y physico-chemical parameters evaluated, including the composition of amino acids, minerals and vitamins, did not allow to determine the effectiveness of yeasts for mass rearing of Anastrepha ludens, A. obliqua and $C$. capitata. However, Nutribio and Lallemand LB12163b yeasts are an alternative for mass rearing of $A$. ludens and $A$. obliqua. The assessment of yeasts in the larval diet also requires experimental bioassays including sexual competition as a variable of response.

Key words: larval diet, protein source, sterile insect technique, mass rearing, Vienna 8 TSL.

reproducción en la fase de adulto (Tsisipis, 1989, Rivera Ciprian et al., 2007). La calidad nutrimental de la dieta está determinada a su vez por la calidad de la fuente de proteína. La deficiencia de ésta repercute en una menor tasa de emergencia en adultos, menor tamaño de las hembras adultas y mayor tiempo de maduración de los oocitos (Kaspi et al., 2002). Además, incrementa la duración de la fase larvaria (Cangussu \& Zucoloto, 1997), entre otros efectos. Al variar la fuente de proteína en la dieta larval se han observado diferencias que pueden ser atribuidas tanto a la calidad como a la cantidad de éstas. La cantidad de proteína requerida en una dieta está influenciada por su calidad nutrimental, y a su vez está determinada por la composición de aminoácidos y por la digestibilidad que presenta (Vanderzant, 1973, Chang, 2004).

La levadura inactiva en polvo es el único ingrediente disponible como fuente de proteína en las dietas larvarias para cría masiva de todas las especies de moscas de la fruta, proporcionando una proteína de alto valor biológico, además de vitaminas del complejo $\mathrm{B}$, carbohidratos y grasas esenciales. La levadura torula [Candida utilis (Henneberg) Lodder \& Kreger Van-Rij] es la más utilizada en diferentes crías masivas de moscas de la fruta (Tanaka et al., 1969, Peleg \& Rhode, 1970, Schwarz et al., 1985, Singh et al., 1988, Chan et al., 1990, Stevens, 1991, Vargas et al., 1994, Moreno et al., 1997, Chang et al., 2001, Artiaga-López et al., 2004). Sin embargo, a nivel experimental, también se ha utilizado la levadura de cerveza (Saccharomyces cerevisiae) (Tzanakakis \& Economopoulos, 1967, Zucoloto, 1987, Salles, 1992, Cangussu \& Zucoloto, 1997, Chang et al., 2004) y caseína (Cangussu \& Zucoloto, 1997, Moreno et al., 1997, Rivera Ciprian, 2007). La levadura es el ingrediente con mayor costo en las crías masivas de moscas de la fruta para la aplicación de la TIE, razón por la cual se buscan 
continuamente alternativas de menor costo, por ejemplo, soya hidrolizada (Chan et al., 1990), proteína hidrolizada y levaduras enriquecidas (Chang, 2009).

Las levaduras secas inactivas existentes en el mercado, de acuerdo con su composición tienen potencial para ser utilizadas en la preparación de dietas para la producción masiva de moscas de la fruta; sin embargo, desde el establecimiento de las crías masivas de A. ludens en 1994 y de A. obliqua en 1997 (Artiaga-López, 2004) se ha utilizado levadura tipo torula correspondiente a una sola marca, lo cual genera una dependencia comercial. De acuerdo con las especificaciones del producto, es posible hipotetizar que, si la levadura cumple con las especificaciones de calidad descrita en los formatos de adquisición del ingrediente utilizado para cría masiva, entonces podrían ser tan eficientes como la levadura de uso común. De acuerdo con lo anterior, el objetivo de este trabajo fue determinar si los parámetros fisicoquímicos y microbiológicos que describen la composición de las levaduras; así como el bioensayo a nivel experimental y semi-masivo permiten determinar su eficiencia para la cría masiva de las moscas de la fruta, A. ludens, A. obliqua y C. capitata cepa Vienna 8 TSL sin inversión cromosómica.

\section{MATERIALES Y MÉTODOS}

Evaluación de Levaduras. Las seis levaduras comerciales evaluadas correspondieron a dos levaduras torula
(Candida utilis): 1) Borregard (Borregard Schweiz AG, Grupo PAUSA, S.A. de C.V., México, D.F.), 2) Lake States (Lake Wausau Paper Lake States, Import \& Export, Inc. Matamoros, Tamps., Méx.). Cuatro levaduras de cerveza (Saccharomyces cerevisiae): 1) Lallemand LBI 2240-25 (Coltec, Guatemala, C.A.), 2) Lallemand LBI 2163 b (Coltec, Guatemala, C.A.), 3) Nutribio (Fermentaciones Mexicanas, S.A. de C.V., SAFMEX, Ixtaczoquitlán, Veracruz, México), y 4) extracto de levadura Nupro (Alltech de México, México) (Cuadros 1,2). La levadura Lake States se consideró como testigo, ya que se ha utilizado por más de 20 en años en la producción masiva de $A$. ludens, $A$. obliqua y $C$. capitata.

Material biológico. El material biológico para las pruebas fue obtenido a partir de huevos recolectados del material producido en la Planta Moscafrut (Programa Moscafrut SAGARPA-IICA, Metapa de Domínguez, Chiapas, México), y Planta Moscamed "El Pino" (Programa regional contra la Mosca del Mediterráneo/USDA-APHIS, Santa Rosa, Guatemala, C.A.). Las moscas fueron criadas bajo las metodologías descritas por Stevens (1991), Artiaga-López et al., (2004) y Cáceres et al., (2000), respectivamente. La cepa utilizada de $C$. capitata correspondió a la Vienna 8 TSL sin inversión.

Diseño Experimental. La evaluación de las levaduras se llevó a cabo en tres etapas. La primera etapa consistió en un análisis de las características fisicoquímicas y microbiológicas de dichas levaduras. La segunda etapa consistió en un bioensayo a nivel experimental. La terce-

Cuadro 1. Análisis fisicoquímico y análisis microbiológico de las levaduras utilizadas en la preparación de las dietas para el desarrollo de larvas de Anastrepha ludens, A. obliqua y Ceratitis capitata cepa Vienna 8 TSL sin inversión en condiciones de cría masiva.

\begin{tabular}{|c|c|c|c|c|c|c|c|}
\hline & Borregard & $\begin{array}{c}\text { Lallemand } \\
\text { LBI } 224025\end{array}$ & $\begin{array}{l}\text { Lallemand } \\
\text { LBI } 2163 \text { b }\end{array}$ & Nupro & Nutribio & Lake & Especificaciones \\
\hline \multicolumn{8}{|c|}{ Análisis fisicoquímico } \\
\hline $\mathrm{Ph}(\mathrm{UI})$ & 6.30 & 6.27 & 6.30 & 4.35 & 5.71 & 5.78 & $5-7$ \\
\hline Acidez (\%) & 0.071 & 0.158 & 0.103 & 0.200 & 0.091 & 0.126 & $0.018-0.304$ \\
\hline Proteína (\%) & 56.06 & 46.24 & 50.30 & 49.77 & 40.54 & 48.33 & $45-55$ \\
\hline \multicolumn{8}{|c|}{ Análisis microbiológico } \\
\hline Cuenta total (UFC/g) & $<10$ & 40 & 100 & 37500 & $<10$ & 500 & 7,000 \\
\hline Hongos (UFC/g) & $<10$ & $<10$ & 20 & 1500 & 10 & $<10$ & $<10$ \\
\hline
\end{tabular}




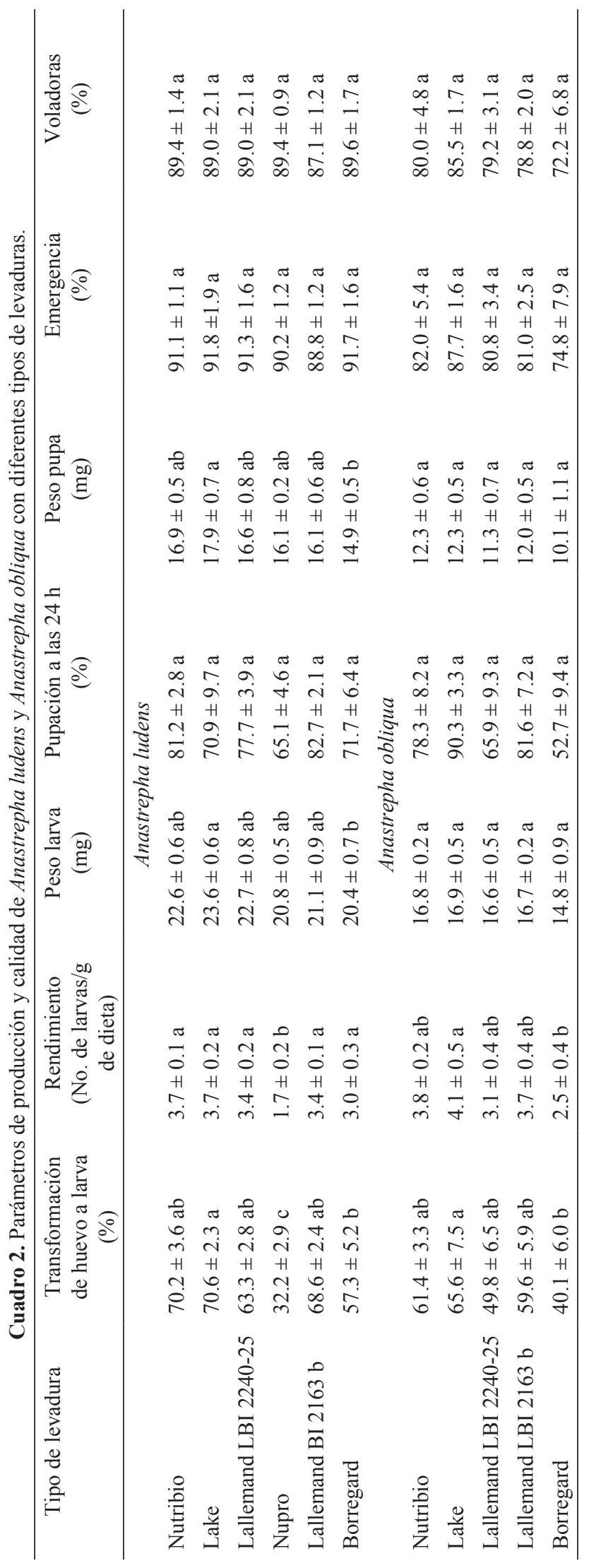


ra etapa correspondió a la evaluación semi-masiva en los respectivos módulos de producción. La evaluación de la calidad y adecuación al uso de las levaduras se realizó de acuerdo a un diseño experimental en bloques al azar, en el cual los tratamientos estuvieron definidos por las marcas de levadura y los bloques correspondieron a los días de preparación de la dieta. Se realizó una repetición por día por cada tratamiento, las cuales se describen en la sección correspondiente.

Caracterización fisicoquímica y microbiológica. Cada levadura fue evaluada con base en las metodologías descritas en los Manuales de Procedimientos de Cría (Muñoz-Barrios et al., 2015, Orozco-Dávila \& Artiaga-López, 2016, Orozco-Dávila et al., 2016) y Control de Calidad de la Planta de Cría y Esterilización de Moscas de la Fruta y Parasitoides (Hernández-Ibarra et al., 2015). Los parámetros de referencia fueron: Humedad (5-8\%), pH (5.57.0), solubilidad (mínimo 31\%), densidad (máximo 0.90 $\mathrm{g} / \mathrm{cm}^{3}$ ), cenizas ( $8 \%$ máximo), proteína $(45-55 \%)$, cuenta total de bacterias (máximo 7,500 UFC), hongos $(<50$ UFC), levaduras ( $<50 \mathrm{UFC})$, y coliformes totales $(<3$ NMP).

Bioensayos. Los bioensayos consistieron en la elaboración de la dieta para las larvas, evaluando en cada etapa los mismos parámetros. Para la evaluación se utilizó un diseño en bloques al azar, en el cual los tratamientos estuvieron definidos por las levaduras mencionadas anteriormente y los bloques por los días en que fue preparado el alimento correspondiente a cada repetición. Las levaduras fueron utilizadas para la elaboración de la dieta larvaria, lo cual se realizó de acuerdo a lo establecido en el Manual de Procedimientos de Cría. Para esto, los ingredientes en seco (polvo de olote 18 y 19\% (Mt. Pulaski Products Inc, Chicago IL), harina de maíz 8.7 y 5.3 \% (Maíz Industrializado del Sureste S.A. de C.V, Arriaga Chiapas, México), azúcar 9 y $8.2 \%$ (Ingenio Huixtla, Huixtla, Chiapas, México), Levadura lake 6.3 y 6\% (Lake States, Div. Rhinelander Paper Co. Rhinelander Wis.), metil p-ben 0.18 y $0.10 \%$ (Mallinckrodt Speciality, Chemicals Co. St. Louis Mis.), benzoato de sodio 0.33 y $0.40 \%$ (Cia. Universal de Industrias, S.A. de C.V., México), goma guar $0.1 \mathrm{y}$ 0.1\% (Tic Gums, Inc Belcamp, Md.), ácido cítrico 0.43 y 0.44\% (Anhidro Acidulantes FNEUM, Mexana S. A. de C.V., Morelos, México), fueron agregados a la mezcladora CRT Modelo CPM-30 (127V, 60Hz, 1100W) (CRT Global S.A. de C.V., Santa Catarina, N. L., México) sin un orden establecido manteniendo las mismas proporciones para cada mezcla, para $A$. obliqua y $A$. ludens, respectivamente. El mezclado de los ingredientes se realizó en seco durante 10 minutos, después se le agregó el agua y se siguió mezclando durante $5 \mathrm{~min}$. Posteriormente se dejó reposar 30 min. Después se verificó la textura para determinar la cantidad de agua necesaria para tener la consistencia adecuada.

La unidad experimental en el bioensayo consistió de una charola $(772 \times 397 \times 73 \mathrm{~mm}$, Model 805208 , Molded Fiber Glass Tray Co., USA) con $7 \mathrm{~kg}$ de dieta sembrada con $1.2 \mathrm{ml}$ de huevo de A. ludens (24200 huevos $/ \mathrm{ml})$, y una charola con $6 \mathrm{Kg}$ de dieta sembrada con $1.5 \mathrm{ml}$ para A. obliqua (16200 huevos/ml). En total se realizaron 12 repeticiones por cada levadura, cada repetición fue considerada como un bloque por prepararse en días diferentes. Las charolas con dieta sembrada se mantuvieron los primeros tres días tapadas con tela tipo pañalina y cubierta con plástico para $A$. obliqua, para el caso de $A$. ludens se cubrieron únicamente con tela para favorecer el desarrollo larvario. Las larvas y pupas fueron mantenidas en cuartos de cría de la planta siguiendo los manuales de procedimientos de sus respectivos módulos de producción en la Planta Moscafrut, y manejadas de acuerdo a lo establecido en los Manuales de Procedimientos de Cría (Orozco-Dávila \& Artiaga-López, 2016, Orozco-Dávila et al., 2016), Control de Calidad de la Planta de Cría y Esterilización de Moscas de la Fruta y Parasitoides (Hernández-Ibarra et al., 2015) y el manual de la FAO/IAEA/USDA (2014).

La unidad experimental en el bioensayo para C. capitata sin inversión consistió en la evaluación a nivel experimental con base en un diseño en bloques al azar, en el cual los tratamientos estuvieron definidos por las levaduras y los bloques por los días en que fueron preparadas las dietas correspondientes a cada una de las 12 repeticiones. Cada repetición consistió en una unidad experimental de una charola $(600 \times 500 \times 100 \mathrm{~mm})$ con $5 \mathrm{~kg}$ de dieta sembrada con $12 \mathrm{ml}$ de huevo de C. capitata (29545 huevos/ $\mathrm{ml}$ ). La dieta se preparó en una mezcladora con capacidad de 18 litros. El contenido de la dieta fue $7.5 \%$ de polvo de olote, 7.5 de bagazo de caña, $4 \%$ de salvado de trigo, $12 \%$ azúcar, 9\% levadura, $1.7 \%$ ácido cítrico, $0.3 \%$ de benzoato de sodio, 0.4 de nipagin, $57.6 \%$ de agua. Las dietas fueron manejadas de acuerdo a lo descrito en los Manuales de procedimientos de la Planta Moscamed (MuñozBarrios et al., 2015).

Evaluación de cría semi-masiva. Las evaluaciones a nivel semi-masivo de $A$. ludens y A. obliqua fueron realizadas únicamente con las levaduras denominadas Nutribio y Lallemand LBI 2163 b, utilizando como control levadura Lake States. Para ambas especies, se seleccionaron las levaduras con las cuales se obtuvieron mayor transforma- 
ción de huevo a larva en los bioensayos. Se realizaron tres repeticiones de una tonelada para A. ludens y A. obliqua. La formulación, preparación y manejo de las dietas se realizó de la misma manera que en el bioensayo y de acuerdo a los manuales operativos de la Planta Moscafrut. La evaluación a nivel semi-masivo de $C$. capitata fue realizada con las levaduras probadas Lake States, Nupro y Lallemand. Se realizaron dos repeticiones, cada una de $300 \mathrm{~kg}$ distribuidos en charolas con $5 \mathrm{~kg}$ de dieta. Para el mezclado de la dieta se utilizó una mezcladora de 1tonelada. Cada repetición se preparó de manera independiente. El manejo de las dietas se realizó de la misma manera que en el bioensayo.

Variables de respuesta. Las variables evaluadas para $A$. ludens y A. obliqua fueron las descritos en los Manuales de Procedimientos de Cría y Control de Calidad de la Planta de Cría y Esterilización de Moscas de la Fruta y Parasitoides (Hernández-Ibarra et al., 2015, Orozco-Dávila \& Artiaga-López, 2016, Orozco-Dávila et al., 2016). Estas fueron: 1) Transformación de huevo a larva, que se determinó con base en el porcentaje de larva recuperada de cada unidad experimental de dieta sembrada con 28,800 y 24,300 huevos por ml para $A$. ludens y A. obliqua, respectivamente. 2) Peso de larvas estimado, considerando tres muestras de un gramo por cada unidad experimental. 3) Rendimiento expresado con base en el número total de larvas recuperadas por cada gramo de dieta. 4) Pupación a las $24 \mathrm{~h}$ estimada utilizando tres muestras de un gramo de pupas, para ello se cuantificaron el número de pupas y se expresó en porcentaje con base en el total de individuos contenidos en la muestra. 5) Peso promedio de la pupa, para su estimación se tomaron tres nuestras de 100 pupas por cada unidad experimental, se pesó cada muestra y se estimó el peso en miligramos. 6) Porcentaje de emergencia pre-irradiación, 7) porcentaje de voladoras pre-irradiación, la emergencia y voladoras fueron determinadas colocando 100 pupas en el fondo de un tubo de plástico negro de $9 \mathrm{~cm}$ de diámetro y $10 \mathrm{~cm}$ de altura, con talco en sus paredes internas y colocado en forma vertical. A los cinco días después de montada la prueba, el contenido del tubo se contabilizó para obtener el número de moscas no emergidas (puparios llenos), moscas medio emergidas (por diferencia se obtiene la emergencia), moscas deformes y moscas no voladoras (para la obtención de moscas voladoras). Se realizaron cinco repeticiones de esta prueba.

Las variables respuesta evaluadas para $C$. capitata correspondieron a: 1) Rendimiento de pupas, fue determinado con base en la cantidad de larva obtenida en cada colecta realizada cada 8 -h, esto refiere a que las larvas de $C$. capitata por tener la capacidad de salir voluntariamente de la dieta cuando terminan su desarrollo son capturadas en charolas que se cambian cada 8-h. La unidad experimental de dieta para $C$. capitata fue sembrada con 354,540 huevos. 2) Peso de pupa, 3) Emergencias y 4) Voladoras se determinaron por cada colecta siguiendo la misma metodología descrita anteriormente para A. ludens y A. obliqua. 5) Mortalidad de adultos bajo estrés a las 48 horas, para ello se colocó una muestra de pupas en el interior de una jaula de plexiglás, las moscas emergidas en un periodo de dos horas desde el inicio de la emergencia fueron colocadas en cajas Petri en un cuarto oscuro a una temperatura de $25 \pm 1^{\circ} \mathrm{C}$, con una humedad de $60-70 \%$ hasta el final de la prueba. Se contabilizaron y retiraron los machos muertos a las 24 horas de iniciada la prueba. A las 48 horas se volvieron a contabilizar los adultos muertos, dando por terminada la prueba. Se realizaron cinco repeticiones por colecta.

Competencia sexual en jaulas de campo. La prueba de competencia sexual de $A$. ludens y A. obliqua se realizó con machos estériles provenientes de cada uno de los alimentos larvarios con las diferentes levaduras (Nutribio, Lallemand LBI 2163 b y Lake States) y con hembras provenientes de la cría de la planta y fértiles. Las jaulas de campo, la preparación del material y el marcaje de los insectos, fue realizado siguiendo los métodos utilizados por Meza-Hernández y Díaz-Fleischer (2006). Para ello, se liberaron 39 machos, siendo 13 machos provenientes de cada alimento y 39 hembras. Las moscas fueron evaluadas con 11 días de edad. El número de apareamientos fue registrado en intervalos de $30 \mathrm{~min}$. Se evaluaron diez jaulas por especie usando dos diferentes cohortes de insectos. Las evaluaciones fueron realizadas de 16:00 a 20:00 h para A. ludens y de 6:00 a 12:00 h para A. obliqua. Estos intervalos, comprende el período de máxima actividad sexual para cada especie (Aluja \& Birke, 1993).

Análisis Estadístico. A los datos obtenidos para cada variable respuesta les fue aplicado el análisis de normalidad y homocedasticidad, en caso de que no se cumplieran los datos fueron transformados a arcoseno. Posteriormente, se realizó Análisis de Varianza y Comparación Múltiple de Medias por el método de Tukey (HSD). Los resultados se analizaron con el programa estadístico JMP versión 5.0.1. Statistical Discovery Software (SAS Institute, 2003). Considerando que el análisis por bloques aleatorios indico que los días de preparación de la dieta no fue un factor significativo $(\alpha=0.05)$, el análisis final se realizó de acuerdo con un diseño completamente al azar. 


\section{RESULTADOS}

\section{Caracterización fisicoquímica y microbiológica}

El análisis fisicoquímico indicó que las levaduras Borregard, Lallemand LBI 2240-25, Lallemand LBI 2163 b y Lake cumplen con las especificaciones establecidas en el Manual de Control de Calidad de la Planta Moscafrut. El extracto de levadura Nupro presentó un $\mathrm{pH}$ de 4.35, quedando fuera de las especificaciones. La levadura Nutribio presentó $40.5 \%$ de proteína quedando por debajo del valor mínimo de referencia (Cuadro 1). El análisis microbiológico determinó para Nupro cargas de 37,500 y 1,500 UFC/ $\mathrm{g}$ para cuenta total y hongos, respectivamente, los cuales son altos en comparación con los valores de referencia de 10,000 y $50 \mathrm{UFC} / \mathrm{g}$, respectivamente. La levadura Lallemand LBI 224025 presentó 9.1 UFC/g para su cuenta de coliformes, el valor de referencia es $<3$ (Cuadro 1).

\section{Bioensayos}

Anastrepha ludens. Los valores mayores de rendimiento $(\mathrm{F}=17.64 ;$ g. $1 .=5,71 ; P<0.001)$ fueron obtenidos con la levadura Lake States. La mayor transformación de huevo a larva $(\mathrm{F}=21.31 ;$ g.l. $=5,71 ; P<0.001)$ fue obtenida con dietas preparadas con levaduras Lake States, Nutribio, Lallemand LBI 2163b, Lallemand LBI 224025 y Borregard. Los valores mayores de peso de la larva $(\mathrm{F}=2.68$; g.l. $=5,71 ; P=0.0291)$ y peso de la pupa $(\mathrm{F}=2.87 ;$ g.l. $=5,71$; $P=0.0208$ ) fueron obtenidos con dietas preparadas con levadura Lake States. La pupación 24 h $(\mathrm{F}=2.26$; g.1. $=5,71$; $P=0.0581)$, emergencia $(\mathrm{F}=0.76 ;$ g.l $=5,71 ; P=0.5757)$ y voladoras $(\mathrm{F}=0.43 ;$ g. $1 .=5,71 ; P=0.8250)$ no presentaron diferencia significativa (Cuadro 2 ).
Anastrepha obliqua. La dieta preparada con la levadura Lake States presentó el mayor rendimiento $(\mathrm{F}=2.90$; g.l. $=4,29 ; P=0.0422)$ y transformación de huevo a larva $(\mathrm{F}=2.90 ;$ g.l. $=4,29 ; P=0.0410)$. Mientras los parámetros de peso de la larva $(\mathrm{F}=2.85 ;$ g.1. $=4,29 ; P=0.0518)$, pupación 24 h $(\mathrm{F}=2.19$; g.l $=4,29 ; P=0.0996)$, peso de la pupa $(\mathrm{F}=1.81 ;$ g.1. $=4,29 ; P=0.1585)$, emergencia $(\mathrm{F}=0.92$; g.1. $=4,29 ; \quad P=0.4652)$ y voladoras $(\mathrm{F}=1.31 ;$ g.1. $=4,29$; $P=0.2964)$ no presentaron diferencias significativas (Cuadro 2).

Ceratitis capitata cepa Vienna 8 TSL sin inversión cromosómica. Los parámetros de producción y calidad presentados en el Cuadro 3 indican que no se observaron diferencias significativas en el rendimiento $(\mathrm{F}=1.46$; g.l. $=5,66 ; P=0.2148)$, emergencia a la segunda $(\mathrm{F}=0.43$; g.l. $=5,66 ; P=0.8221)$ y tercera colecta $(\mathrm{F}=1.43 ;$ g.l. $=5,66$; $P=0.2286)$, voladoras en la segunda $(\mathrm{F}=0.65 ;$ g.l. $=5,66$; $P=0.6625)$ y tercera colecta $(\mathrm{F}=0.11 ; \mathrm{g} .1 .=5,66 ; P=0.9889)$ y mortalidad en la segunda $(\mathrm{F}=0.53 ;$ g.l. $=5,66 ; P=0.7504)$ y tercera colecta $(\mathrm{F}=1.52 ;$ g.1. $=5,66 ; P=0.1931)$. El peso de pupa presentó diferencias significativas, los valores mayores correspondieron a la pupa obtenida de dieta preparada con las levaduras Lake States, Nutribio, Lallemand LBI2163b y Nupro, los valores menores correspondieron a las levaduras Lallemand LBI 2240-25 y Borregard $(\mathrm{F}=7.25$; g.l. $=5,66 ; P<0.0001)$.

\section{Evaluación de cría semi-masiva}

El contenido de vitaminas y el perfil de aminoácidos de las muestras de levaduras utilizadas para la evaluación a nivel semi-masivo en la elaboración de dietas para el desarrollo larvario de A. ludens, A. obliqua y C. capitata se describen en los Cuadros 4 y 5 .

Cuadro 3. Parámetros de producción y calidad de Ceratitis capitata cepa Vienna 8 TSL sin inversión con diferentes tipos de levaduras.

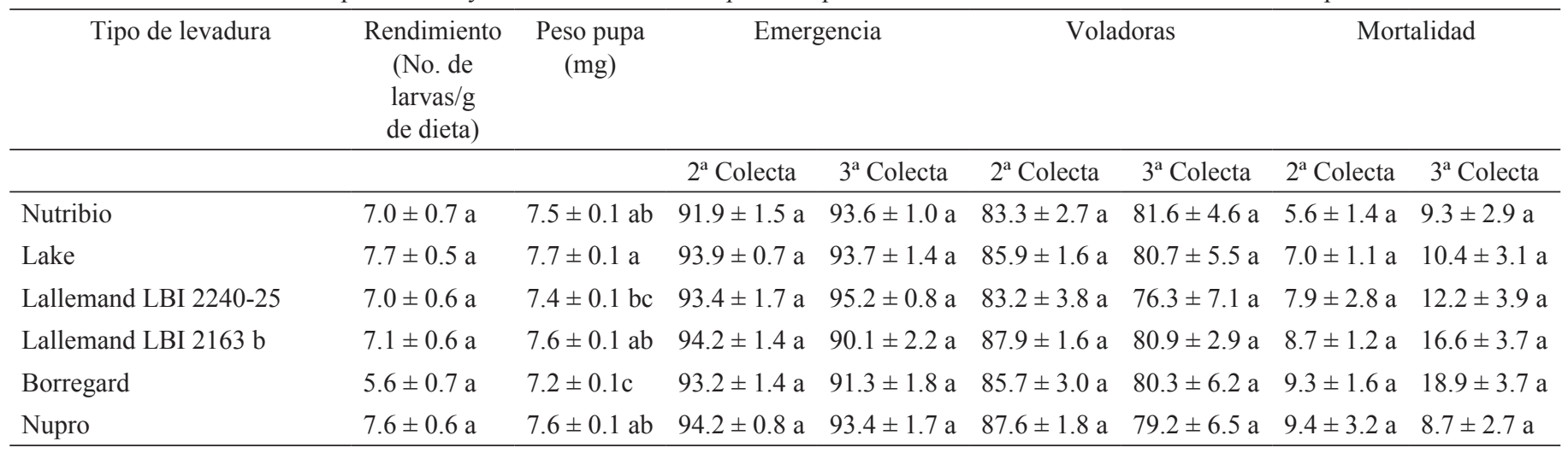


Cuadro 4. Contenido de vitaminas en muestras de levaduras utilizadas para la elaboración de dietas para el desarrollo de larvas de Anastrepha ludens, A. obliqua y Ceratitis capitata cepa Vienna 8 TSL sin inversión en condiciones de cría masiva.

\begin{tabular}{|c|c|c|c|}
\hline Tipo de vitamina & Lallemand LBI 2163 b & Nutribio & Lake \\
\hline \multicolumn{4}{|c|}{ Vitamina } \\
\hline Niacina $(\mathrm{mg} / 100 \mathrm{~g})$ & $49.93 \pm 1.040$ & $46.29 \pm 0.21$ & $38.70 \pm 0.33$ \\
\hline Piridoxina(mg/100g) & $0.59 \pm 0.0120$ & $1.42 \pm 0.061$ & $0.65 \pm 0.014$ \\
\hline Ac. Fólico( $\mu \mathrm{g} / 100 \mathrm{~g})$ & ND & ND & ND \\
\hline Vitamina C(mg/100g) & $0.325 \pm 0.002$ & $0.281 \pm 0.005$ & $0.018 \pm 0.0001$ \\
\hline Vitamina A(mg/100g) & $2.84 \pm 0.080$ & $3.83 \pm 0.10$ & $2.75 \pm 0.08$ \\
\hline Vitamina D(mg/100g) & $17.26 \pm 0.870$ & $1.88 \pm 0.005$ & $2.22 \pm 0.11$ \\
\hline Vitamina E(mg/100g) & $0.100 \pm 0.003$ & $0.32 \pm 0.008$ & $0.36 \pm 0.004$ \\
\hline Hierro & $22.81 \pm 0.15$ & $3.68 \pm 0.007$ & $13.78 \pm 0.55$ \\
\hline \multicolumn{4}{|c|}{ Proteína y humedad } \\
\hline Proteína & $42.34 \pm 0.89$ & $47.50 \pm 0.95$ & $45.73 \pm 0.11$ \\
\hline Humedad & $2.09 \pm 0.03$ & $2.78 \pm 0.08$ & $2.84 \pm 0.01$ \\
\hline
\end{tabular}

$\mathrm{ND}=$ No detectado.

Cuadro 5. Contenido de aminoácidos en muestras de levaduras utilizadas para la elaboración de dietas para el desarrollo de larvas de Anastrepha ludens, A. obliqua y Ceratitis capitata cepa Vienna 8 TSL sin inversión en condiciones de cría masiva.

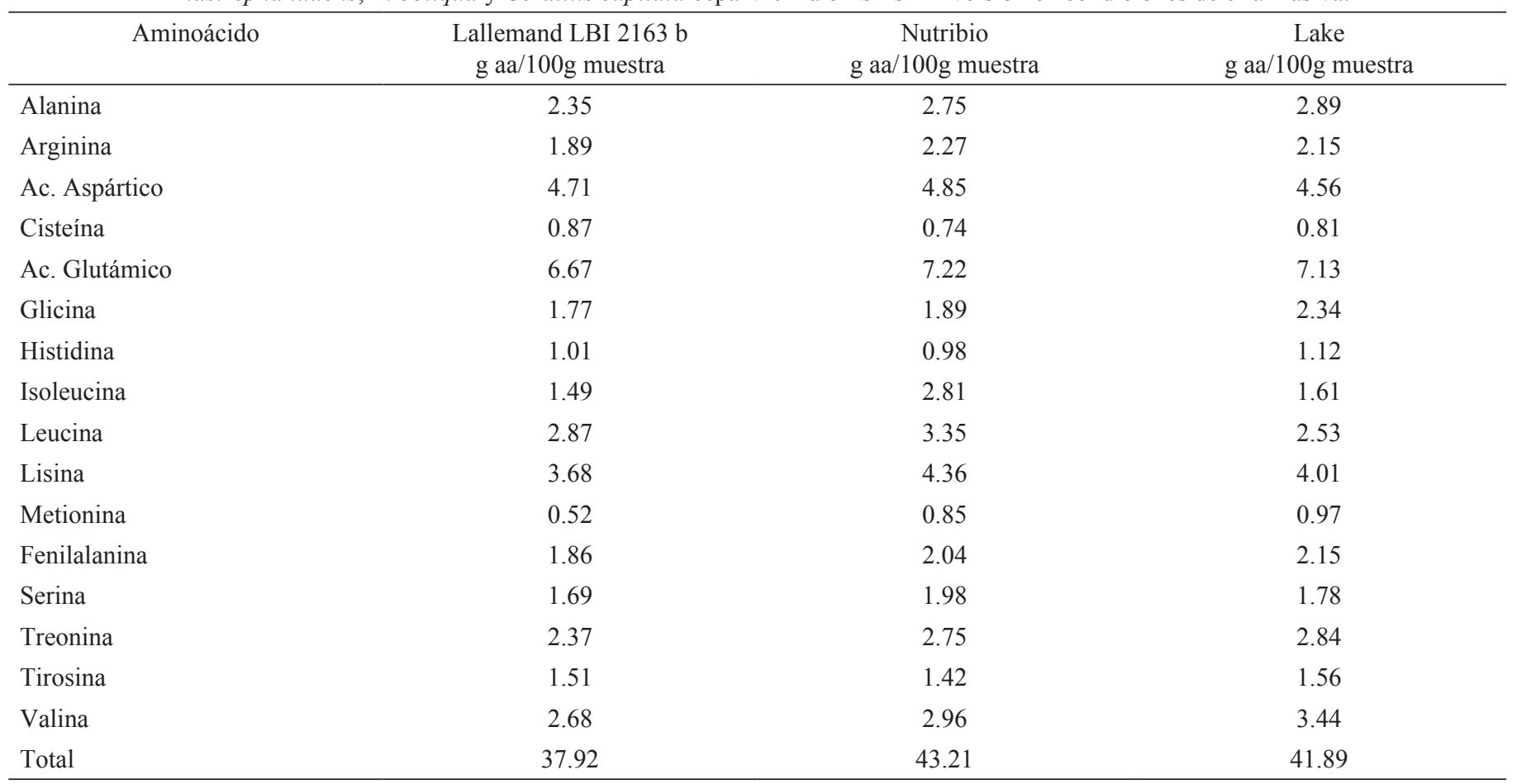


Anastrepha ludens. Los resultados en producción y calidad indicaron que sólo el rendimiento no presentó diferencia significativa $(\mathrm{F}=2.18$, g.1. $=2,8 ; P=0.1943)$. Los valores mayores de transformación de huevo a larva $(\mathrm{F}=10.42$, g.l. $=2,8 ; P=0.0112)$, peso de la larva $(\mathrm{F}=15.21$, g.l. $=2,8 ; P=0.0045)$, peso de la pupa $(\mathrm{F}=15.58$, g.l. $=2$, $8 ; P=0.0042)$, emergencia $(\mathrm{F}=6.95$, g.l. $=2,8 ; P=0.0274)$ $\mathrm{y}$ voladoras $(\mathrm{F}=5.54, \mathrm{~g} .1 .=2,8 ; P=0.0432)$ fueron obtenidos con la dieta preparada con la levadura Lallemand LB12163b. Mientras que los valores mayores de pupación 24 h $(\mathrm{F}=303.09$, g.l. $=2,8 ; P<0.0001)$ fueron obtenidos con la dieta preparada con levadura Lake States (Cuadro 6). Las dietas preparadas con levadura Lake States espolvorearon y no se presentó oxidación en la superficie. La larva presentó buen tamaño y menor dieta en el tracto digestivo durante la recuperación. Esto favoreció la formación de pre-pupas que se vio reflejado en los porcentajes de pupación. No se observó presencia de grumos en el alimento lo que facilitó la recuperación en húmedo y seco. Las dietas preparadas con levadura Nutribio presentaron larvas de buen tamaño, aunque con presencia de alimento en el tracto digestivo, y durante la recuperación larvaria se observó alimento larvario oxidado, humedad y con presencia de grumos, dificultando la recuperación. Se observó menor formación de pre-pupas que influyeron en los bajos porcentajes de pupación. Las dietas preparadas con levadura Lallemand LB12163b presentaron larvas con alimento en su tracto digestivo cuando fueron separadas de la dieta; además, exhibieron baja humedad, oxidación en la parte superior y en el interior, dieta totalmente espolvoreada. No se observó un desfase larvario importante. Sin embargo, se observó alimento en el tracto digestivo de la larva pero con menor cantidad que la Nutribio. El tamaño de la larva fue homogéneo. Durante la recuperación se observó menos grumos en el alimento larvario en comparación con la Nutribio y la separación larvaria no presentó dificultad ni en húmedo ni en seco.

Anastrepha obliqua. Los valores mayores de rendimiento $(\mathrm{F}=9.98$, g.l. $=2,8 ; P=0.0124)$, transformación de huevo a larva $(\mathrm{F}=10.23$, g.l. $=2,8 ; P=0.0116)$ y pupación $24 \mathrm{~h}$ $(\mathrm{F}=22.14$, g.l. $=2,8 ; P=0.0017)$ fueron obtenidos con la dieta preparada con levadura Lake States. Los valores mayores de peso de la larva $(\mathrm{F}=8.65$, g.1. $=2,8 ; P=0.0171)$, peso de la pupa $(\mathrm{F}=4.65$, g.l. $=2,8 ; P=0.0603)$, emergencia $(\mathrm{F}=4.14, \mathrm{~g} .1 .=2,8 ; P=0.0743)$ y voladoras $(\mathrm{F}=2.10$, g.l. $=2,8 ; P=0.2035)$ fueron obtenidos con la dieta preparada con la levadura Lallemand LB12163b (Cuadro 6). Las dietas preparadas con levadura Lake States presentaron buenas características sin presencia de oxidación y grumos. Se observaron larvas de buen tamaño sin desfase larvario y no se presentó mortalidad. La separación en húmedo fue fácil y se observó formación de pre-pupas que favorecieron la pupación. Las dietas elaboradas con levadura Nutribio se oxidaron y presentaron desfase larvario. Se observó alimento en el tracto digestivo de la larva. Durante la recuperación se observaron grumos, lo que dificultó un poco la separación en húmedo. Las dietas preparadas con levadura Lallemand LB12163b presentaron larvas con el tracto digestivo limpio, no se observaron larvas muertas y el alimento larvario espolvoreó más que la dieta con levadura Nutribio. No se presentaron problemas durante la separación.

Ceratitis capitata cepa Vienna 8 TSL sin inversión cromosómica. Los resultados a nivel masivo indicaron que el peso de larva $(\mathrm{F}=5.13 ; \mathrm{gl}=2,12 ; P=0.0246)$, peso de pupa $(\mathrm{F}=10.01 ; \mathrm{gl}=2,12 ; P=0.0028)$ y voladoras $(\mathrm{F}=6.79$;

Cuadro 6. Parámetros de producción y calidad de Anastrepha ludens y Anastrepha obliqua con diferentes tipos de levaduras.

\begin{tabular}{|c|c|c|c|c|c|c|c|}
\hline Tipo de levadura & $\begin{array}{c}\text { Transformación } \\
\text { de huevo a larva } \\
(\%)\end{array}$ & $\begin{array}{c}\text { Rendimiento } \\
\text { (No. de larvas/g } \\
\text { de dieta) }\end{array}$ & $\begin{array}{l}\text { Peso larva } \\
\quad(\mathrm{mg})\end{array}$ & $\begin{array}{c}\text { Pupación } 24 \\
\text { horas }(\%)\end{array}$ & $\begin{array}{l}\text { Peso pupa } \\
\quad(\mathrm{mg})\end{array}$ & $\begin{array}{c}\text { Emergencia } \\
(\%)\end{array}$ & $\begin{array}{c}\text { Voladoras } \\
\text { pre-irradiación } \\
(\%) \\
\end{array}$ \\
\hline \multicolumn{8}{|c|}{ Anastrepha ludens } \\
\hline Nutribio & $77.53 \pm 0.85 b$ & $3.56 \pm 0.01 \mathrm{a}$ & $23.29 \pm 0.29 b$ & $51.28 \pm 0.11 \mathrm{c}$ & $17.40 \pm 0.14 b$ & $94.22 \pm 1.03 \mathrm{~b}$ & $93.05 \pm 1.12 \mathrm{a}$ \\
\hline Lake & $78.57 \pm 0.04 \mathrm{ab}$ & $3.55 \pm 0.01 \mathrm{a}$ & $23.89 \pm 0.07 \mathrm{~b}$ & $87.20 \pm 1.67 \mathrm{a}$ & $18.03 \pm 0.13 \mathrm{a}$ & $97.00 \pm 0.39 \mathrm{ab}$ & $96.44 \pm 0.38 \mathrm{a}$ \\
\hline $\begin{array}{l}\text { Lallemand } \\
\text { BI } 2163 \text { b }\end{array}$ & $80.66 \pm 0.01 \mathrm{a}$ & $3.50 \pm 0.03 \mathrm{a}$ & $24.51 \pm 0.50 \mathrm{a}$ & $64.53 \pm 0.99 b$ & $18.49 \pm 0.13 \mathrm{a}$ & $97.39 \pm 0.29 \mathrm{a}$ & $96.05 \pm 0.67 \mathrm{a}$ \\
\hline \multicolumn{8}{|c|}{ Anastrepha obliqua } \\
\hline Nutribio & $85.33 \pm 1.12 \mathrm{~b}$ & $4.33 \pm 0.01 \mathrm{~b}$ & $18.26 \pm 0.03 \mathrm{~b}$ & $81.75 \pm 2.64 \mathrm{~b}$ & $13.50 \pm 0.01 \mathrm{a}$ & $86.56 \pm 0.78 \mathrm{a}$ & $81.73 \pm 0.99 \mathrm{a}$ \\
\hline Lake & $90.96 \pm 0.72 \mathrm{a}$ & $4.46 \pm 0.03 \mathrm{a}$ & $18.67 \pm 0.08 \mathrm{a}$ & $96.28 \pm 0.61 \mathrm{a}$ & $13.32 \pm 0.06 \mathrm{a}$ & $89.20 \pm 1.15 \mathrm{a}$ & $84.44 \pm 1.60 \mathrm{a}$ \\
\hline $\begin{array}{l}\text { Lallemand } \\
\text { LBI } 2163 \mathrm{~b}\end{array}$ & $90.33 \pm 1.75 a$ & $4.34 \pm 0.02 b$ & $18.73 \pm 0.20 \mathrm{a}$ & $94.25 \pm 1.01 \mathrm{a}$ & $13.49 \pm 0.05 \mathrm{a}$ & $89.79 \pm 0.42 \mathrm{a}$ & $84.83 \pm 0.74 \mathrm{a}$ \\
\hline
\end{tabular}


$\mathrm{gl}=2,12 ; P=0.0106)$ presentaron diferencias significativas. Mientras que el rendimiento $(\mathrm{F}=2.03 ; \mathrm{gl}=2,12$; $P=0.1730)$, transformación de huevo a larva $(\mathrm{F}=2.28$; $\mathrm{gl}=2,12 ; \quad P=0.1452), \quad$ emergencia $\quad(\mathrm{F}=3.03 ; \quad \mathrm{gl}=2,12$; $P=0.0860)$ y mortalidad $(\mathrm{F}=1.48 ; \mathrm{gl}=2,12 ; P=0.2666)$ no presentaron diferencias significativas (Cuadro 7).

\section{Competencia sexual en jaulas de campo}

Anastrepha ludens. Los resultados del estudio en jaulas de campo no indicaron efecto significativo de las levaduras Lallemand LB12163b y Nutribio con respecto al control en el desempeño sexual de los machos estériles con hembras de cría fértiles $(\mathrm{gl}=2,27 ; \mathrm{F}=3.275 ; \mathrm{P}<0.05)$.

Anastrepha obliqua. Se observó una diferencia significativa entre la levadura Nutribio y el control (gl=2,27; $\mathrm{F}=3.275 ; \mathrm{P}<0.05$ ). Entre la levadura Lallemand LB12163b $\mathrm{y}$ control no se observaron diferencias significativas $(\mathrm{gl}=2,27 ; \mathrm{F}=4.95 ; \mathrm{P}<0.05)$ (Fig. 1).

\section{DISCUSIÓN}

Los análisis de los parámetros fisicoquímicos y microbiológicos de las levaduras evaluadas mostraron que la solubilidad (\%), densidad ( $\mathrm{g} / \mathrm{cc})$, contenido de células de levadura (UFC/g), coliformes (UFC/g) y la actividad de células vivas son características de las levaduras secas inactivas evaluadas en este experimento que se cumplieron para todas las muestras de acuerdo con los valores establecidos en los manuales de control de calidad para el uso en la dieta para el desarrollo de larvas de moscas de la fruta. Sin embargo, la levadura Lallemand LB12163b estuvo fuera de especificación para el porcentaje de humedad; la Nupro en el pH (UI), cuenta total (UFC/g) y contenido de hongos (UFC/g) y Nutribio en el contenido de proteína (\%). A pesar de que estas levaduras estuvieron fuera de especificación, se decidió no descartarlas para el bioensayo, considerando que los valores de referencia han sido determinados con base en el historial del ingrediente y no por las necesidades o efecto sobre las larvas de las especies de mosca de la fruta consideradas en este trabajo. Además, no existen estudios sobre los niveles de tolerancia/ susceptibilidad cuando alguno de los parámetros está fuera de lo especificado. Por otro lado, los resultados obtenidos por el bioensayo indicaron que las levaduras Lallemand LB12163b y Nutribio fueron la segunda y tercera con mayor eficiencia después de la Lake States considerada como testigo para A. ludens y A. obliqua. Mientras que para $C$. capitata cepa Vienna 8 TSL sin inversión fueron la levadura Lallemand LB12163b y Nupro, después del testigo Lake States. La eficiencia de estas levaduras, las cuales no fueron descartadas a nivel semi-masivo quedo confirmada por las pruebas de competencia sexual, las cuales indicaron que para el caso de A. ludens el mayor número de cópulas se obtuvo con moscas criadas en levadura Nutribio; mientras que para $A$. obliqua fue con las levaduras Lallemand LB12163b y Lake States. Lo anterior indica que los análisis fisicoquímicos y microbiológicos que se realizan actualmente en las Plantas Moscafrut y Moscamed a la levadura como fuente de proteína en la dieta larvaria no permiten determinar si ésta posee o no la calidad adecuada para el desarrollo de las larvas, por lo que es necesaria la realización de bioensayos.

En nuestro trabajo consideramos como alternativa el 'valor biológico' de la fuente proteína, el cual se refiere a la composición y disponibilidad de los aminoácidos (Chan et al., 1990, Chang, 2009), además del contenido de vitaminas y minerales. Sin embargo, los resultados indicaron el mismo problema que para los análisis fisicoquímicos y microbiológicos, es decir, no permiten identificar diferencias significativas que puedan explicar que empleando la levadura Nutribio, las moscas de A. obliqua produjeron el menor valor de apareamientos, mientras que las moscas de $A$. ludens presentaron mayor eficiencia en el desarrollo y número de apareamientos en comparación con las levaduras Lake States y Lallemand LB12163b. De acuerdo con ello y con lo observado por Chang (2009), las diferencias en los parámetros de desarrollo y calidad

Cuadro 7. Parámetros de producción y calidad de Ceratitis capitata cepa Vienna 8 TSL sin inversión con diferentes tipos de levaduras.

\begin{tabular}{|c|c|c|c|c|c|c|c|}
\hline Tipo de levadura & $\begin{array}{c}\text { Transformación } \\
\text { de huevo a larva } \\
(\%)\end{array}$ & $\begin{array}{c}\text { Rendimiento } \\
\text { (No. de larvas/g } \\
\text { de dieta) }\end{array}$ & $\begin{array}{l}\text { Peso larva } \\
\quad(\mathrm{mg})\end{array}$ & $\begin{array}{l}\text { Peso pupa } \\
\quad(\mathrm{mg})\end{array}$ & $\begin{array}{c}\text { Emergencia } \\
(\%)\end{array}$ & $\begin{array}{c}\text { Voladoras } \\
(\%)\end{array}$ & Mortalidad \\
\hline Lake & $15.62 \pm 0.99 a$ & $12.03 \pm 0.77 \mathrm{a}$ & $10.15 \pm 0.17 \mathrm{a}$ & $7.70 \pm 0.09 a$ & $88.83 \pm 9.86 a$ & $85.52 \pm 0.99 b$ & $14.30 \pm 2.02 \mathrm{a}$ \\
\hline Nupro & $11.31 \pm 1.33 \mathrm{a}$ & $8.89 \pm 1.01 \mathrm{a}$ & $10.00 \pm 0.16 \mathrm{ab}$ & $7.54 \pm 0.16 b$ & $89.77 \pm 0.87 a$ & $88.47 \pm 0.22 \mathrm{a}$ & $11.25 \pm 2.06 \mathrm{a}$ \\
\hline Lallemand BI 2163 b & $13.75 \pm 1.85 \mathrm{a}$ & $10.59 \pm 1.42 \mathrm{a}$ & $9.48 \pm 0.14 b$ & $6.94 \pm 0.12 b$ & $91.52 \pm 0.60 \mathrm{a}$ & $88.96 \pm 0.70 \mathrm{a}$ & $16.32 \pm 2.20 \mathrm{a}$ \\
\hline
\end{tabular}



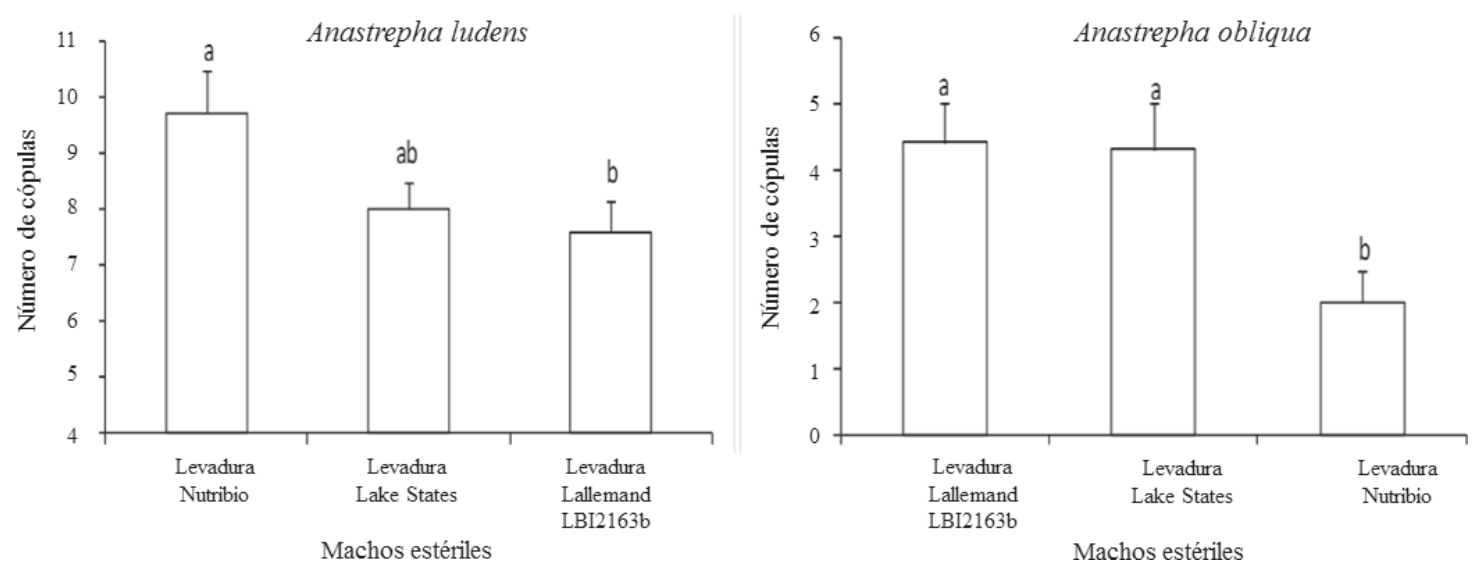

Figura 1. Número de cópulas realizadas por machos desarrollados en diferentes levaduras con hembras silvestres obtenidas de mango infestado.

de las moscas criadas en diferentes tipos de levadura no se pueden explicar únicamente por las diferencias en la composición de aminoácidos, minerales y vitaminas, sino que es necesario determinar su disponibilidad y relación funcional con la fisiología del insecto para describir cómo su deficiencia o adquisición a través de la alimentación o por procesos de síntesis afectan el desarrollo, crecimiento, mantenimiento de tejidos, reproducción, demanda de energía y la tasa de mortalidad (Genc, 2006). Nuestros datos indican que el contenido de las levaduras probadas, aunque con algunas variaciones contienen los mismos aminoácidos; sin embargo, se requiere determinar si existen diferencias en su disponibilidad y digestibilidad, es decir los aminoácidos digestibles constituyen una estimación más adecuada del nitrógeno disponible, ya que el nitrógeno total sobreestima el valor proteico aprovechable e implica que su uso puede llevar a una formula no balanceada (Perdomo et al., 2004). Algunos trabajos han destacado que la ausencia de algunos aminoácidos, entre ellos la alanina, ácido aspártico, cistina, ácido glutámico, glicina, prolina, serina, tirosina, causan atraso en el desarrollo de larva (Chang, 2004), lo cual puede reflejarse en un bajo porcentaje de pupación a las $24 \mathrm{~h}$. En contraste, cuando la dieta no contiene arginina, isoleucina, leucina, lisina, histidina, metionina, fenilalanina, treonina, triptófano y valina, la larva muere (Chang, 2004), generando una baja transformación de huevo a larva o bajo rendimiento. El triptófano es necesario para la formación de pigmentos visuales, el glutamato es neurotransmisor y la prolina es necesaria para el desarrollo y como fuente de energía (Genc, 2006) que podrían afectar el desempeño sexual. Además de los aminoácidos, se debe considerar el contenido de vitaminas. La Niacina (vitamina $B_{3}$ ) y la nia- cinamida son precursores de los nucleótidos de piridina y su ausencia o limitación resulta en la mortalidad de las larvas, reducción del porcentaje de pupación e incremento del tiempo de pupación, así como en la disminución de la emergencia de C. capitata (Jang, 1986). La efectividad de la Niacina depende del contenido de tiamina, riboflavina, ácido pantoteíco, piridoxina, ácido fólico, biotina, inositol, colina, cloro y ácido p-aminobenzoico (Chang \& Li, 2004, Braga Sobrinho et al., 2006).

\section{CONCLUSIONES}

De acuerdo con los resultados se rechaza la hipótesis de que una levadura eficiente para la cría masiva de las moscas de la fruta Anastrepha ludens, A. obliqua y C. capitata cepa Vienna 8 TSL sin inversión cromosómica es aquella que cumple con las especificaciones de calidad descritas por los análisis microbiológicos y fisicoquímicos, incluyendo la composición de aminoácidos, minerales y vitaminas. La determinación de la eficiencia de una levadura requiere de la realización de un bioensayo a nivel experimental que incluya como variable respuesta la competencia sexual. Se considera urgente la determinación de los parámetros que permitan asegurar la calidad de las levaduras utilizadas actualmente en las crías masivas de las moscas de la fruta.

AGRADECIMIENTOS. Este proyecto fue ejecutado con apoyo económico del Programa Moscafrut SAGARPA-IICA. Los autores manifiestan su agradecimiento al personal de los Departamentos de Producción, Control de Calidad, Almacén de Ingredientes y Administrativo de la Plantas Moscamed y Moscafrut; así como al personal del 
Departamento de Colonización y Cría de la Subdirección de Desarrollo de Métodos por su apoyo en la ejecución de este proyecto.

\section{LITERATURA CITADA}

Aluja, M., \& Birke, A. (1993). Habitat use by adults of Anastrepha obliqua (Diptera: Tephritidae) in a mixed mango and tropical plum orchard. Annals of the Entomological Society of America, 86, 799-812.

Artiaga-López, T., Hernández, A., Domínguez-Gordillo J., Moreno D. S., \& Orozco-Dávila, D. (2004). Mass-production of Anastrepha obliqua at the Moscafrut Fruit Fly Facility, Mexico,pp 389-392. In: B. N. Brian [Ed.]. Proceedings of the $6^{\text {th }}$ International Symposium on Fruit Fly of Economic Importance. 6-10 May 2002, Heriotdale, Johannesburg, South Africa.

Braga Sobrinho, R., Caceres, C., Islam, A., Wornoaypporn, V., \& Enkerlin, W. (2006). Diets based on soybean protein for Mediterranean fruit fly. Pesquisa Agropecuária Brasileira, 41, 705-708.

Cáceres, C., Fisher, K., \& Rendón, P. (2000). Mass rearing of the Medfly temperature sensitive letal genetic sexing strain in Guatemala, pp. 551-558. In: K.H. Tan [Ed.]. Area-Wide Control of Fruit Flies and Other Insect Pests, Penerbit Universiti Sains Malaysia, Penang.

Caceres, C., Hendrichs, J., \& Vreysen, M. J. B. (2014). Development and improvement of rearing techniques for fruit flies (Diptera: Tephritidae) of economic importance. International Journal of Tropical Insect Science, 34, S1: S1-S12.

Cangussu, J. A., \& Zucoloto, F. S. (1997). Effect of protein sources on fecundity, food acceptance, and sexual choice by Ceratitis capitata (Diptera: Tephritidae). Revista Brasileira de Biología, 57, 611- 618.

Chan, Jr., H. T., Hansen, J. D., \& Tam, S. Y. T. (1990). Larval diets from different protein sources for Mediterranean fruit flies (Diptera: Tephritidae). Journal of Economic Entomology, 83, 1954-1958.

Chang, C. L., Albrecht, C. P., \& Kurashima R. (2001). Adult reproductive capacity of Ceratitis capitata (Diptera: Tephritidae) on a chemically defined diet. Annals of the Entomological Society of America, 94, 702-706.

Chang, C. L. (2004). Effect of amino acids on larvae and adult of Ceratitis capitata (Diptera: Tephritidae). Annals of the Entomological Society of America, 97, 529-535.

Chang, C. L. (2009). Evaluation of yeast and yeast products in larval and adult diets for the oriental fruit fly, Bactrocera dorsalis, and adult diets for the medfly, Ceratitis capitata, and the melon fly, Bactrocera curcurbitae. 9 pp. Journal of Insect Science. 9, 23, available online: insectscience.org/9.23

Chang, C. L., \& Li, Q. X. (2004). Dosage effects between dietary niacin and other B vitamins on larval development of Ceratitis capitata (Diptera:Tephritidae). Annals of the Entomological Society of America, 97, 536-540.

Enkerlin, W. R. (2005). Impact of fruit fly control programmes using the sterile insect technique, pp. 651-676. In: V.A. Dyck, J. Hendrichs and A.S. Robinson [Eds.]. Sterile Insect Technique. Principles and Practice in Area-Wide Integrated Pest Management. IAEA. Springer. Netherlands.
FAO/IAEA/USDA. (2014). Product Quality Control for Sterile MassReared and Released Tephritid Fruit Flies. Joint FAO/IAEA Programme of Nuclear Techniques in Food and Agriculture. Manual Version 6.0. IAEA, Vienna 8, Austria, 164 pp. Available at: http:// www-naweb.iaea.org/nafa/ipc/public/sterile-mass-reared-v6.pdf

Genc, H. (2006). General principles of insect nutritional ecology. Trakya University Journal of Natural Sciences, 71, 53-57.

Hendrichs, J., Ortiz, G., Liedo, P., \& Schwarz A. (1983). Six years of successful medfly program in Mexico and Guatemala, pp. 353365. In: R. Cavalloro [Ed.], Proceedings Symposium: Fruit Flies of Economic Importance. CEC/IOBC. International Symposium, 16-19 November 1982, Athens Greece. A. A. Balkena, Rotterdam, The Netherlands.

Hendrichs, J. (1996). Action programs against fruit flies of economic importance: session overview, pp. 513-519. In: B. A. McPheron and G. J. Steck [Eds.]. Fruit Fly Pests. A World Assessment of Their Biology and Management. St Lucie Press, Delray Beach, FL.

Hernández, E, Pedro Rivera, J., \& Artiaga-López, T. (2014). Generic larval diet for mass-rearing. three species of Anastrepha (Diptera: Tephritidae). Journal of Tropical Insect Science, doi: 10.1017/S1742758414000046.

Hernández-Ibarra, M. D. R., Bravo-López, J. J., Reyna-Cigarroa, C. A., Coutiño-Molina, J. C., Villatoro-Telles, R., \& MezaArriaga, D. D. (2015). ITCC01 - Instructivo de Trabajo del Laboratorio de Control de Calidad del Proceso de Anastrepha spp. Dirección General de Sanidad Vegetal/ Dirección del Programa Nacional de Moscas de la Fruta/ Subdirección de Producción Moscafrut. 2016. Secretaría de Agricultura, Ganadería, Desarrollo Rural, Pesca y Alimentación. Servicio Nacional de Sanidad, Inocuidad y Calidad Agroalimentaria. Metapa de Domínguez, Chiapas. $72 \mathrm{pp}$.

Jang, E. B. (1986). Effects of niacin deficiency on growth and development of the Mediterranean fruit fly (Diptera: Tephritidae). Journal of Economic Entomology, 79, 558-561.

Kaspi, R., Mossinson, S., Drezner, T., Kamensky, B., \& Yuval, B. (2002). Effects of larval diet on development rates and reproductive maturation of male and female Mediterranean fruit flies. Physiological Entomology, 27, 29-38.

Meza-Hernández, J. S., \& Díaz-Fleischer, F. (2006). Comparison of sexual compatibility between laboratory and wild Mexican fruit flies under laboratory and field conditions. Journal of Economic Entomology, 99, 1979-1986.

Moreno, D. S., Ortega-Zaleta, D.A., \& Mangan. R. L. (1997). Development of artificial larval diets for West Indian fruit fly (Diptera: Tephritidae). Journal of Economic Entomology, 90, 427-434.

Muñoz-Barrios, R., Silva Villarreal, L.C., \& González Bravo, P. I. (2015). Manual de Cría y Esterilización de Machos de Ceratitis capitata (Wiedemann) Cepa TSL. Dirección General de Sanidad Vegetal/ Dirección del Programa Nacional de Moscas de la Fruta/ Subdirección de Producción Moscamed. Secretaría de Agricultura, Ganadería, Desarrollo Rural, Pesca y Alimentación. Servicio Nacional de Sanidad, Inocuidad y Calidad Agroalimentaria. Metapa de Domínguez, Chiapas. 24 pp.

Orozco-Dávila, D., \& Artiaga-López, T. (2016). MPPMAO01 Manual para la Cría Masiva de Anastrepha obliqua (Macquart). Dirección General de Sanidad Vegetal/ Dirección del Programa 
Nacional de Moscas de la Fruta/ Subdirección de Producción Moscafrut. Secretaría de Agricultura, Ganadería, Desarrollo Rural, Pesca y Alimentación. Servicio Nacional de Sanidad, Inocuidad y Calidad Agroalimentaria. Metapa de Domínguez, Chiapas. $23 \mathrm{pp}$.

Orozco-Dávila, D., Domínguez, J., Reyes, J., Villaseñor, A., \& Gutiérrez, J. M. (2004). SIT and biological control of Anastrepha fruit flies in Mexico, pp. 245-249. In: B. N. Brian [Ed.]. Proceedings of the $6^{\text {th }}$ International Symposium on Fruit Flies of Economic Importance. 6-10 May 2002, Heriotdale, Johannesburg, South Africa.

Orozco-Dávila, D., Solís-Echeverría, E.A., Gamboa, G.E., BaézMartínez, R., \& González-López, I. (2016). MPPMAL01 - Manual de Procedimientos para la Cría Masiva de la Mosca Mexicana de la Fruta Anastrepha ludens (Loew). Dirección General de Sanidad Vegetal/ Dirección del Programa Nacional de Moscas de la Fruta/ Subdirección de Producción Moscafrut. Secretaría de Agricultura, Ganadería, Desarrollo Rural, Pesca y Alimentación. Servicio Nacional de Sanidad, Inocuidad y Calidad Agroalimentaria. Metapa de Domínguez, Chiapas. 23 pp.

Peleg, B. A., \& Rhode, R. H. (1970). New larval medium and improved pupal recovery method for Mediterranean fruit fly in Costa Rica. Journal of Economic Entomology, 63, 1319-1321.

Perdomo, M. C., Vargas J., R. E., \& Campos G., J. (2004). Valor nutritivo de la levadura de cervecería (Saccharomyces cerevisiae) y de sus derivados, extracto y pared celular, en la alimentación aviar. Archivos Latinoamericanos de Producción Animal, 12, 8995.

Rivera Ciprian, J. P., Hernández, E., Toledo, J., Salvador, M., \& Silva, R. (2007). Dieta Texturizada con agar para el desarrollo larvario de tres especies de moscas de la fruta (Diptera: Tephritidae). Folia Entomológica Mexicana, 46, 37-52.

Reyes F., J., Santiago M., G., \& Hernández M., P. (2000). The Mexican fruit fly eradication program, pp. 377-380. In: K.H. Tan [Ed.]. Area-Wide Control of Fruit Flies and Other Insect Pests, Penerbit Universiti Sains Malaysia, Penang.
Salles L., A. B. (1992). Metodologia de cria de Anastrepha fraterculus (Wied. 1830) (Diptera: Tephritedae) em dieta artificial em laboratorio. Annais da Sociedade Entomológica do Brasil, 21, 479-487.

SAS Institute. (2003). JMP Statistical, Discovery Software, Version 5.0.1. SAS Institute Inc., Carg., North Carolina.

Schwarz G. A., Zambada, A., Orozco, D., Zavala, J. L., \& Calkins, C. O. (1985). Mass rearing of the Mediterranean fruit fly at Metapa, México. Florida Entomologist, 68, 467-477.

Singh, P., Leppla, N. C., \& Adams, F. (1988). Feeding behavior and dietary substrates for rearing larvae of the Caribbean fruit fly, Anastrepha suspensa. Florida Entomologist, 71, 380-384.

Stevens, L. (1991). Manual of Standard Operating Procedures (SOP) for the Mass-Rearing and Sterilization of the Mexican Fruit Fly, Anastrepha ludens (Loew). USDA-APHIS, South Central Region, Míssion Texas. 39 p.

Tanaka, N., Steiner, L. F., Ohinata, K., \& Okamoto, R. (1969). Low cost larval rearing medium for mass production of oriental and Mediterranean fruit flies. Journal of Economic Entomology, 63, 967-968.

Tzanakakis, M. E., \& Economopoulos, A. P. (1967). Two efficient larval diets for continuous rearing of the olive fruit fly. Journal of Economic Entomology, 60, 660-663.

Tsisipis, J. A. (1989). Nutrition, pp. 103-119. In: Robinson A. S. (Ed.). Fruit flies their biology, natural enemies and control. Volume 3B. Elsevier Press. Amsterdam.

Vanderzant, E. S. (1973). Improvement in the rearing diet for Chisopha carnea and the aminoacid requeriment for growth. Journal of Economic Entomology, 66, 366-338

Vargas, R. I., Mitchell, S., Hsu, C., \& Walsh, W. A. (1994). Laboratory evaluation of diets of processed corncob, torula, yeast, and wheat germ on four developmental stages of Mediterranean fruit fly (Diptera: Tephritidae). Journal of Economic Entomology, 87, 91-95.

Zucoloto F. S. (1987) Feeding habits of Ceratitis capitata: can larvae recognize a nutritionally effective diet? Journal of Insect Physio$\log y, 33,349-353$. 